\section{M1/M2 polarisation state of M-CSF blood- derived macrophages in systemic sclerosis}

We read with interest the work conducted by Soldano and colleagues, ${ }^{1}$ exploring M1/M2 macrophage surface markers in circulating blood cells in systemic sclerosis (SSc). Using a wide phenotypic characterisation of circulating cells by flow cytometry, the authors highlight that the percentage of CD14+ cells coexpressing M2 markers (CD206, CD163 and CD204) and M1 markers (TLR4) is higher in patients with SSc than in healthy subjects (HSs). Similarly, CD204+ circulating cells coexpressing M2 markers (CD163, CD206) and M1 markers (TLR4, CD80, CD86) were also over-represented in patients with SSc. These results strengthen the concept of a specific macrophage signature in $\mathrm{SSc}$ which goes beyond the dichotomous M1/M2 paradigm..$^{2-4}$ However, this study was only conducted on undifferentiated circulating blood cells and the validation of their results on differentiated macrophages is still to be determined.

In support of Soldano's results, ${ }^{1}$ we present here, a phenotypic analysis conducted on macrophage-colony stimulating factor (M-CSF) resting blood monocyte-derived macrophages (MDM) from glucocorticoid (GC)-free patients fulfilling ACR/EULAR 2013 classification criteria for SSc in comparison with HSs, evaluating the mean of fluorescence intensity (MFI) ratio of six polarisation markers (CD80, CD206, CD204, CD163, CD169 and CD200R1) and supporting the existence of a mixed M1/M2 signature in SSc MDM. In parallel, to assess the value of these markers as 'polarisation markers' in this model of MDM, MDM from HSs were also polarised in vitro into M1 (IFNy+LPS), M2a (IL-4 and IL-13) and M2c (IL-10+ dexamethasone). ${ }^{56}$

Our results confirmed, in HS MDM, the higher expression of CD80 and CD169 in polarised M1 in comparison with M2 and unpolarised/resting M0 subtypes (figure 1A). On the contrary, CD200R1 and CD206 were only overexpressed in HS M2a. The expressions of CD163, usually considered as an M2 marker and of CD204, were significantly higher in HS M2a when compared with HS M1 but were not different from those of HS M0 (figure 1A). ${ }^{6}$ In SSc M0, CD200R1 and CD204 were significantly decreased in comparison with $\mathrm{M} 0$ from HS (figure 1B). The expression of these markers was comparable in SSc M0 and HS M1 (respectively in SSc M0 and HS M1, MFI ratio for CD200R1: $1.83 \pm 0.11$ vs $1.64 \pm 0.096 ; \mathrm{p}>0.05$ and
A

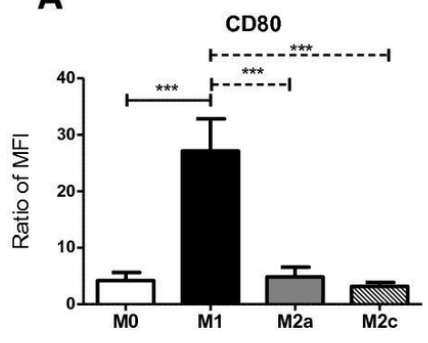

CD200R1

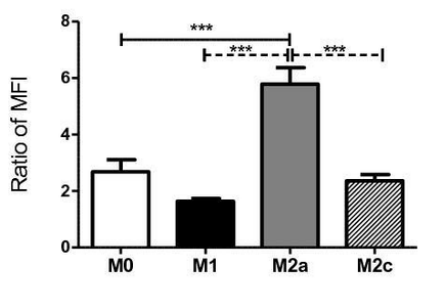

CD163

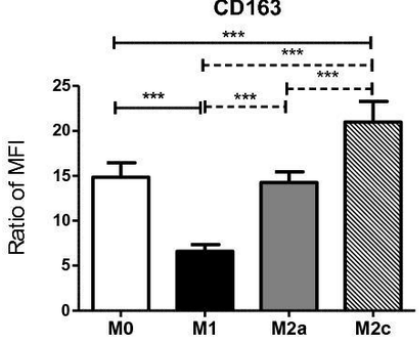

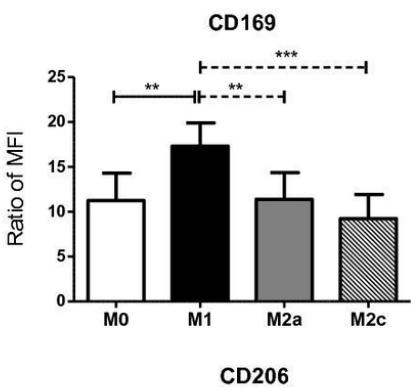
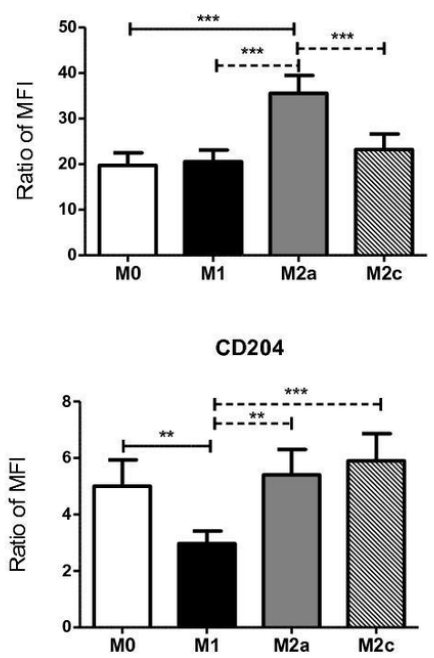

B

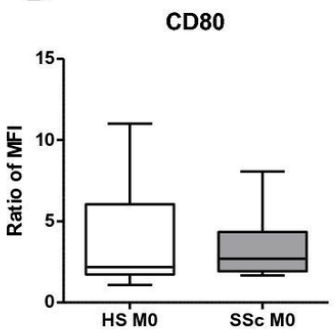

CD200R1
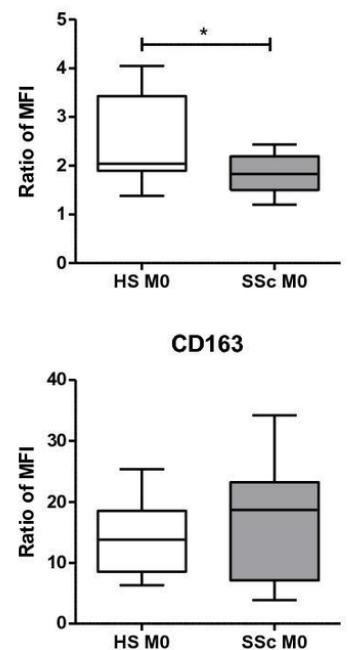
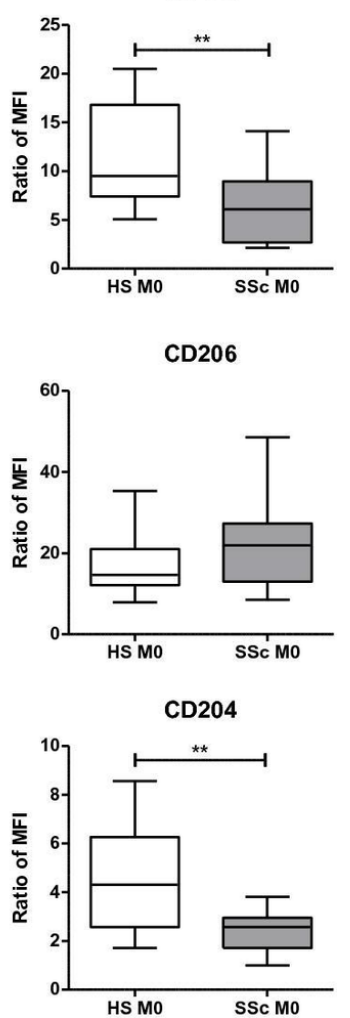

Figure 1 Polarisation markers of M-CSF MDMs from patients with SSC and HSs. (A) Primary human blood monocytes from HSs were differentiated into MDM in vitro in the presence of $50 \mathrm{ng} / \mathrm{mL}$ of M-CSF for 6 days. At day 6 , media were replaced by a fresh media (RPMI with $5 \%$ of fetal bovine serum with $10 \mathrm{ng} / \mathrm{mL}$ of M-CSF) and MDM were polarised for additional 24 hours by the addition of $20 \mathrm{ng} / \mathrm{mL}$ IFNy and $20 \mathrm{ng} / \mathrm{mL}$ LPS (M1), by 20

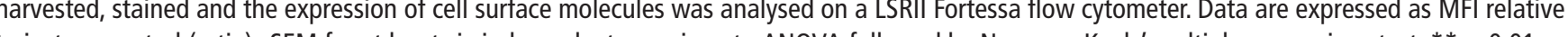
to isotype control (ratio) \pm SEM for at least six independent experiments. ANOVA followed by Newman-Keuls' multiple comparison test, ${ }^{* *} \mathrm{p}<0.01$ and . (B) Primary human blood monocytes from HSs and patients with SSC were differentiated into MDM in vitro in the presence of $50 \mathrm{ng} / \mathrm{mL}$ hours. These M0 unpolarised/resting cells were then harvested, stained and the expression of cell surface molecules was analysed on a LSRII Fortessa flow cytometer. Data are expressed as MFI relative to isotype control (ratio) \pm SEM for at least 13 HSs and for 11-16 patients with SSc. Student unpaired t-test, ${ }^{*} \mathrm{p}<0.05$ and ${ }^{*} \mathrm{*} p<0.01$. ANOVA, analysis of variance; HSs, healthy subjects; MDM, monocyte-derived macrophages; MFI, mean fluorescence intensity; SSC, systemic sclerosis. 
MFI ratio for CD204: $2.45 \pm 0.24$ vs $2.97 \pm 0.44 ; p>0.05)$. By contrast, the expression of CD169 was significantly decreased in SSc M0 when compared with both HS M0 (figure 1B) and HS M1 (SSc M0 vs HS M1, MFI ratio for CD169: 6.20 1.23 vs $17.31 \pm 2.56 ; \mathrm{p}<0.001)$. The expression of this marker was comparable in SSc M0 and HS M2c (SSc M0 vs HS M2c: MFI ratio for CD169: $6.20 \pm 1.23$ vs 9.21 \pm 2.71 ; $p>0.05)$.

Therefore, in this phenotypic analysis based on MFI, M0 MDM from patients with SSc showed a specific phenotype expressing some markers in the same way as M1 and other markers similarly to M2 or unpolarised M0 macrophages. Within a different approach, this result supports the existence of a mixed M1/M2 signature in SSc MDM, as suggested by Soldano et al concerning subpopulations of circulating cells. ${ }^{1}$ Our results on marker expressions among polarised MDM in vitro also alert on the value of so-called M1 or M2 markers, as CD163 may be considered as an M2a marker in comparison with M1 but is similar to M0 resting macrophages (figure 1A). CD163 appears to be more specific to dexamethasone-induced $\mathrm{M} 2 \mathrm{c}$ in comparison with $\mathrm{M} 2 \mathrm{a}$ (figure $1 \mathrm{~A}$ ), a result that could be especially important when considering patients with GCs. CD163 could even be induced in M1 when dexamethasone is added to the medium. ${ }^{57}$ These results also bring light to the results from Soldano's figure 2B, pointing the increased percentage of CD163+/CD206+ among CD204+cells in patients with SSc with GCs in comparison with both patients with SSc without GCs and HSs. ${ }^{1}$

Acknowledging that the small sample size of our data represents a limitation for our conclusions, we agree with Soldano's suggestion to think outside the box: beyond the dichotomous M1/M2 paradigm, using new phenotyping approaches may offer a more encompassing vision of macrophages in SSc. ${ }^{4-68-10}$

\section{Alain Lescoat, ${ }^{1,2}$ Alice Ballerie, ${ }^{1,2}$ Stephane Jouneau, ${ }^{1,3}$ Olivier Fardel, ${ }^{1,4}$ Laurent Vernhet, ${ }^{1}$ Patrick Jego, ${ }^{1,2}$ Valérie Lecureur ${ }^{1}$ \\ ${ }^{1}$ Univ Rennes, CHU Rennes, Inserm, EHESP, Irset (Institut de recherche en santé, environnement et travail), Rennes, France \\ ${ }^{2}$ Department of Internal Medicine, Rennes University Hospital, Rennes, France ${ }^{3}$ Department of Respiratory Diseases, Rennes University Hospital, Rennes, France ${ }^{4}$ Pôle Biologie, Rennes University Hospital, Rennes, France}

Correspondence to Dr Alain Lescoat, Department of Internal Medicine, Rennes University Hospital, Rennes 35000, France; alain.lescoat@chu-rennes.fr

Handling editor Josef S Smolen

Contributors All authors contributed to the draft, writing and experiments presented in this correspondence.

Funding This work was supported by the "Groupe Francophone de la Recherche sur la Sclérodermie"(GFRS).
Competing interests None declared.

\section{Patient consent Obtained.}

Ethics approval The ethics committee approval was obtained from our local ethics committees (Committees for protection of persons (CPP) Ouest V France, CPP approval No: 2015-A01221-48; study N.15/26-988).

Provenance and peer review Not commissioned; internally peer reviewed.

(c) Author(s) (or their employer(s)) 2019. No commercial re-use. See rights and permissions. Published by BMJ.

$A L$ and $A B$ contributed equally.

\section{Check for updates}

To cite Lescoat A, Ballerie A, Jouneau S, et al. Ann Rheum Dis 2019;78:e127.

Received 24 August 2018

Accepted 30 August 2018

Published Online First 29 September 2018

\section{SLinked}

http://dx.doi.org/10.1136/annrheumdis-2018-214371

Ann Rheum Dis 2019;78:e127. doi:10.1136/annrheumdis-2018-214333

\section{REFERENCES}

1 Soldano S, Trombetta AC, Contini P, et al. Increase in circulating cells coexpressing M1 and M2 macrophage surface markers in patients with systemic sclerosis. Ann Rheum Dis 2019:77:1842-5.

2 Mosser DM, Edwards JP. Exploring the full spectrum of macrophage activation. Nat Rev Immunol 2008;8:958-69.

3 Moreno-Moral A, Bagnati M, Koturan S, et al. Changes in macrophage transcriptome associate with systemic sclerosis and mediate GSDMA contribution to disease risk. Ann Rheum Dis 2018;77:596-601.

4 Behmoaras J, Petretto E. Cell function in disease: there are more than two parties at play. Ann Rheum Dis 2019;78:e20.

5 Murray PJ, Allen JE, Biswas SK, et al. Macrophage activation and polarization: nomenclature and experimental guidelines. Immunity 2014;41:14-20.

6 Chávez-Galán L, Olleros ML, Vesin D, et al. Much More than M1 and M2 macrophages, there are also CD169(+) and TCR(+) Macrophages. Front Immunol 2015;6:263.

7 Tedesco S, Bolego C, Toniolo A, et al. Phenotypic activation and pharmacological outcomes of spontaneously differentiated human monocyte-derived macrophages. Immunobiology 2015;220:545-54

8 Christmann RB, Sampaio-Barros P, Stifano G, et al. Association of Interferonand transforming growth factor $\beta$-regulated genes and macrophage activation with systemic sclerosis-related progressive lung fibrosis. Arthritis Rheumatol 2014:66:714-25

9 Taroni JN, Greene CS, Martyanov V, et al. A novel multi-network approach reveals tissue-specific cellular modulators of fibrosis in systemic sclerosis. Genome Med 2017;9:27

10 Martinez FO, Gordon S. The M1 and M2 paradigm of macrophage activation: time for reassessment. F1000Prime Rep 2014;6:13. 